\title{
Molecular characterisation and antibiotic sensitivity profile of Pasteurella multocida isolated from poultry farms in Malaysia
}

\author{
Mohammad A. Sabsabia ${ }^{a}$ Zunita Zakaria ${ }^{\text {b,c }}$, Jalila Abua, Nik M. Faiz ${ }^{\text {a,c* }}$
}

\begin{abstract}
Fowl cholera has caused significant economic losses in many poultry producing countries worldwide. In Malaysia, outbreaks of fowl cholera are frequently reported and encountered in different types of poultry productions. The objective of this study was to characterise 13 avian Pasteurella multocida, isolated from fowl cholera outbreaks in Central Peninsular Malaysia in the period between 2000 and 2018. The isolates were subjected to multiplex polymerase chain reaction (PCR) for capsular serotyping, disc diffusion method for antimicrobial susceptibility profiles, and molecular genotyping using pulsed-field gel electrophoresis (PFGE) and multilocus sequence typing (MLST). The capsular serotyping showed all 13 Pasteurella multocida isolates belonging to capsular serotype A. The antimicrobial susceptibility showed several multidrug resistance strains among the $P$. multocida isolates. All the isolates were resistant to erythromycin (100\%), streptomycin (68\%), tetracycline (37\%), enrofloxacin (37\%), florfenicol (23\%), penicillin G (14\%), gentamicin (14\%), and amoxicillin (14\%). The PFGE analysis clustered the isolates into three clones. Group A included isolates with a similarity of $87 \%$ from the year 2000, 2013, and 2018. Three sequence types were identified using MLST typing namely, ST129, ST231, and ST355. The ST355 was assigned for the first time in the Rural Industries Research and Development Corporation (RIRDC) database. Besides, ST129 has been reported in India, China, and Sri Lanka, which highlights the possibility of transmission between Asian countries. This study provides an insight into epidemiological information of Pasteurella multocida that causes fowl cholera outbreaks in the central region of Peninsular Malaysia.

Key words: antimicrobial susceptibility, MLST, Pasteurella multocida, PFGE.
\end{abstract}

\section{INTRODUCTION}

Pasteurella multocida (P. multocida) is a Gram-negative bacterium that can cause a wide range of diseases in animals, such as fowl cholera in poultry, haemorrhagic septicemia in bovine, and atrophic rhinitis in swine (Wilkie et al 2012, Wilson and Ho 2013). Fowl cholera is an epizootic, highly contagious avian disease that could affect several avian species including commercial chickens (Botzler 1991). Since it was discovered, it has caused significant economic losses in the poultry industry worldwide (Harper et al 2006). The disease may occur as an acute septicaemia form with high morbidity and mortality (up to $100 \%$ ), or as a localised chronic infection (Heddleston et al 1964, Harper et al 2006). Sudden death for a large number of birds is usually the first clinical sign in acute fowl cholera (Glisson et al 2013). Pasteurella multocida can currently be subdivided into four subspecies: subsp multocida, subsp gallicida, subsp septica, and subsp tigris. All subspecies, excluding tigris, have been isolated from fowl cholera outbreaks (Harper et al 2006). Serotype A is the dominant serotype of $P$. multocida causing fowl cholera while serotypes $\mathrm{B}, \mathrm{D}$, and $\mathrm{F}$ have been less reported to cause disease in poultry (Wilkie et al 2012). Outbreaks

Received: 08.10.2021.

Accepted: 19.01.2021.

aDepartment of Veterinary Clinical Studies, Faculty of Veterinary Medicine, Universiti Putra Malaysia, Selangor, Malaysia.

${ }^{b}$ Department of Veterinary Pathology \& Microbiology, Faculty of Veterinary Medicine, Universiti Putra Malaysia, Selangor, Malaysia.

'Institute of Bioscience, Universiti Putra Malaysia, Selangor, Malaysia.

*Corresponding author: NM Faiz; nikmdfaiz@upm.edu.my were reported in Asia and all over the world (Wang et al 2013, Jones et al 2013, Singh et al 2013). Antibiotics are widely used in the treatment of $P$. multocida infections in poultry, which have increased antibiotic resistance (Murray 1992). A study conducted in Brazil showed $19.64 \%$ of $P$. multocida strains isolated from chicken and turkey farms were multidrug-resistant to three or more drugs in different categories using the disc diffusion method (Furian et al 2016). A number of epidemiological studies were conducted to investigate the distribution of $P$. multocida strains in several countries (Sarangi et al 2016, Li et al 2018, Peng et al 2018). Pulsed-field gel electrophoresis (PFGE) is a genotyping technique that analyses bacterial chromosomes using restriction enzyme into DNA fragments. The PFGE fragments pattern can be used to study the strain variation and evolution (Gunawardana et al 2000). PFGE also has been used to study outbreaks of fowl cholera in poultry (Kardos and Kiss 2005, Sellyei et al 2017). However, multilocus sequence typing (MLST) is the current gold standard typing method for P. multocida, which uses seven housekeeping genes to characterise and study the global distribution of P. multocida sequence types (STs) (Kardos and Kiss 2005, Subaaharan et al 2010, Singh et al 2013).

In Malaysia, avian P. multocida outbreaks were frequently reported in commercial and backyard farms (Arumugam et al 2011, Nafizah et al 2014, Khoo et al 2017). However, information on P. multocida serogroups, antibiotic resistance profile, and molecular genotyping are poorly investigated. Therefore, the aim of this study is to molecular characterise P. multocida isolates from fowl cholera outbreaks submitted to the Laboratory of Bacteriology at Faculty of Veterinary Medicine, Universiti Putra Malaysia, Malaysia. The isolates undergo multiplex 
PCR serotyping to determine the serogroup as well as the disc diffusion method to determine the resistance profile of the isolates. Additionally, genotyping was performed using PFGE and MLST to study the variation and evolution of the P. multocida isolates.

\section{MATERIAL AND METHODS}

\section{BACTERIAL ISOLATES}

Thirteen P. multocida subspecies multocida isolates from the Bacteriology Laboratory, Faculty of Veterinary Medicine, Universiti Putra Malaysia, Malaysia were analysed in this study (table 1). The samples were submitted for diagnostic purposes, between the years 2000 to 2018 from fowl cholera outbreaks in backyard chicken farms located in Selangor, Malaysia. The bacteria isolates were obtained from chicken internal organs including liver, spleen, and lungs, and were subject to biochemical identification. The samples were cultured onto $5 \%$ blood agar (OXOID, UK). Suspected colonies showing $P$. multocida colony morphology, were subjected to biochemical tests, namely, oxidase, indole, Triple Sugar Iron (TSI), Sulfide Indole Motility (SIM), citrate, urease reactions and Ornithine Decarboxylase Test (ODC), trehalose, mannitol, D-sorbitol, and dulcitol.

\section{MOLECULAR IDENTIFICATION}

Genomic DNA was extracted using the boiling method. The bacteria were boiled at $97^{\circ} \mathrm{C}$ for $10 \mathrm{~min}$, then placed in an ice container for $5 \mathrm{~min}$, then centrifugated for 10 $\mathrm{min}$ at room temperature. The isolates were confirmed as $P$. multocida using PCR targeting the KMT1 gene as described by Townsend et al (1998). P. multocida ATCC 12945 was used as a positive control.

\section{MULTIPLEX PCR CAPSULAR TYPING}

The isolates were subjected to capsular serotyping using the primers designed by Townsend et al (2001). Genomic DNA was extracted using the boiling method. The multiplex PCR was carried out with a final reaction volume of $50 \mu \mathrm{l}, 30 \mathrm{PCR}$ cycles; $95^{\circ} \mathrm{C}$ for $45 \mathrm{sec}, 56.2^{\circ} \mathrm{C}$ for $45 \mathrm{sec}, 72^{\circ} \mathrm{C}$ for $45 \mathrm{sec}$. The PCR product was visualised using a UV transilluminator.

\section{ANTIMICROBIAL SUSCEPTIBILITY TEST}

Antimicrobial susceptibility test was carried out using the disc diffusion method following the Clinical and Laboratory Standards Institute standards (CLSI) VET01- A4 (4 ${ }^{\text {th }}$ ed.) and the M45 ( $3^{\text {rd }}$ ed.). Two replicates were performed for each isolate against eight antibiotics, namely, streptomycin $10 \mu \mathrm{g}$, amoxicillin $10 \mu \mathrm{g}$, tetracycline $30 \mu \mathrm{g}$, gentamicin $10 \mu \mathrm{g}$, erythromycin $15 \mu \mathrm{g}$, penicillin $\mathrm{G} 10 \mathrm{u}$, enrofloxacin $5 \mu \mathrm{g}$, and florfenicol 30 $\mu \mathrm{g}$. The bacteria suspensions were cultured on Mueller-Hinton agar then the antibiotic where placed on the plates. After 24 hours of incubation, the average zone of inhibition was measured and interpreted. The Escherichia coli ATCC25922 and Staphylococcus aureus ATCC 29213 were used as quality control.

Table 1. Antibiotic resistance profile for each P. multocida isolated from fowl cholera outbreaks in poultry farms in Malaysia in the period of 2000 to 2018. Pasteurella multocida isolated from fowl cholera outbreaks in poultry farms in Malaysia in the period of 2000 to 2018.

\begin{tabular}{cccccc}
\hline Number & Year & Sample & PFGE & ST & Antibiotics resistance profile ${ }^{\ddagger}$ \\
\hline 1 & 2000 & PM201 & A3 & 129 & E \\
2 & 2000 & PM202 & A3 & - & E \\
3 & 2000 & PM203 & A3 & - & E \\
4 & 2000 & PM204 & A3 & - & E \\
5 & 2013 & PM205 & A1 & 129 & ST, ENR, TE, E \\
6 & 2013 & PM206 & A2 & - & ST, ENR, TE, E. AMX, CN, P \\
7 & 2014 & PM207 & B & 129 & ST, ENR, TE, E, AMX, CN, P, FFC \\
8 & 2016 & PM208 & C1 & 355 & ST, E \\
9 & 2016 & PM209 & C2 & - & ST, E \\
10 & 2016 & PM210 & C3 & - & E \\
11 & 2016 & PM211 & C3 & - & ST, ENR, TE, E, FFC \\
12 & 2018 & PM212 & A1 & - & ST, ENR, TE, E, FFC \\
13 & 2018 & PM213 & A1 & 231 &
\end{tabular}

\$) List of antibiotics the isolates were resistant against, according to M45 ( $3^{\text {rd }}$ ed.) and VET01- A4 ( $4^{\text {th }}$ ed.) of the CLSI standard. ST= Streptomycin, $\mathrm{AMX}=$ Amoxicillin, $\mathrm{TE}=$ Tetracycline, $\mathrm{CN}=$ Gentamicin, $\mathrm{E}=$ Erythromycin, $\mathrm{P}=$ Penicillin $\mathrm{G}, \mathrm{ENR}=$ Enrofloxacin, $\mathrm{FFC}=\mathrm{Florfenicol}$. 
PULSED-FIELD GEL ELECTROPHORESIS (PFGE)

A single colony from each isolate was cultured in brain heart infusion broth (BHI) (OXOID, UK) and incubated at $37^{\circ} \mathrm{C}$ for $24 \mathrm{hr}$. The culture was then mixed to cell suspension buffer and adjusted to 0.6-0.7 of McFarland standard. The cell mixtures were pipetted into CHEF disposable plug moulds (Bio-Rad Laboratories, USA) and allowed to solidify for $10 \mathrm{~min}$ at $4{ }^{\circ} \mathrm{C}$. The plugs were transported into $2 \mathrm{ml}$ cell lysis buffer and incubated in a water bath at $56^{\circ} \mathrm{C}$ for $17 \mathrm{hr}$ with one hour shaking at $100 \mathrm{rpm}$. The plugs were washed two times with deionised water for 10 min each at $50{ }^{\circ} \mathrm{C}$, followed by 5 times washing with the TE buffer. A small slice of each plug was placed in a $2 \mathrm{ml}$ tube containing $200 \mu \mathrm{l}$ per-restriction mixture for $15 \mathrm{~min}$ at $37^{\circ} \mathrm{C}$. Then, a restriction mixture containing the ApaI enzyme was added and incubated at $37^{\circ} \mathrm{C}$ for 2 hr. Finally, the restriction mixture was removed, and 0.5 TBE buffer was added for 5-10 min. The electrophoresis was performed using the following conditions; initial switch time $1 \mathrm{sec}$, final switching time $40 \mathrm{sec}$, a constant voltage of $6 \mathrm{~V}$, and an angle of 120 . With a total running time of $23 \mathrm{hr}$ and $14^{\circ} \mathrm{C}$ running temperature. BioNumerics 6.6 software was used to analyse the P. multocida PFGE profiles. The dendrogram was created with $1 \%$ optimization and $1 \%$ tolerance using the Dice similarity coefficient, by unweighted paired group method of arithmetic averages (UPGMA). Strains with more than three differences in DNA fragments and a similarity of $<85 \%$ were classified into different PFGE types (Van Belkum et al 2007).

\section{MULTILOCUS SEQUENCE TYPING}

One isolate representing each year was characterised via the MLST scheme. Briefly, PCR amplification was carried out for seven housekeeping genes, using the primers designed by Subaaharan et al (2010), then the sequences were analysed using the $P$. multocida MLST RIRDC database $^{1}$ to get the $P$. multocida sequence type (ST).

\section{RESULTS AND DISCUSSION}

Pasteurella multocida is a significant pathogen that causes epizootic diseases in animals including fowl cholera in poultry. Outbreaks of fowl cholera have been reported all over the world in both wild and domestic birds (Botzler 1991, Kardos and Kiss 2005). To enhance our understanding of the variation and transmission of the bacterial strains, many techniques were used to study the population structure of $P$. multocida worldwide (Gunawardana et al 2000, Subaaharan et al 2010). In this study, thirteen isolates confirmed to be $P$. multocida were found to belong to capsular serotype A.

1 http://pubmlst.org/pmultocida_rirde/
The isolates showed an overall high level of antibiotic resistance, five isolates were multidrug resistance (MDR) (PM205, PM206, PM207, PM212, and PM213), as shown in table 1. Also, the PM207 isolate was found to be resistant to all antibiotics tested, namely erythromycin, streptomycin, tetracycline, enrofloxacin, florfenicol, penicillin $\mathrm{G}$, gentamicin, and amoxicillin, which belonged to six classes of antibiotics. MDR strains of P. multocida have been reported in the USA, Germany, Spain, and recently reported in China MDR strains of $P$. multocida against florfenicol, chloramphenicol, ofloxacin tetracycline streptomycin, kanamycin, and sulfamethoxazole (Li et al 2018; Zhu et al 2020). Overall, the isolates were resistances to erythromycin $(100 \%)$, streptomycin (68\%), tetracycline (37\%), enrofloxacin (37\%), florfenicol (23\%), penicillin G (14\%), gentamicin (14\%), and amoxicillin (14\%). Similar findings were reported in a study of avian $P$. multocida in Mississippi, whereby the isolates were resistant $78 \%$ against erythromycin, $78 \%$ streptomycin, and $46 \%$ penicillin (Jones et al 2013). In this study, the high resistance against erythromycin and streptomycin was reported. It is high likely that these occur due to excessive usage of antibiotics in poultry farms in Malaysia. In addition, these antibiotics are also used as growth promoter in poultry feeds in Malaysia (Hassali et al 2018). Besides, P. multocida isolates may also have acquired resistance genes from other Gram-negative bacteria (Wilson and Ho 2013).

The PFGE phylogenetic tree clustered the isolates into three groups shown in table 1. Group A has an $87.1 \%$ similarity among isolates from different years $(2000,2013$, and 2018). Group B showed $85 \%$ similarity, including isolates from the year 2016. PFGE type $\mathrm{C}$ has one isolate that belongs to the year 2014, as shown in figure 1. The high similarity of $87.1 \%$ in group A suggests that these isolates were isolated from related outbreaks of the same strains of P. multocida and had developed or acquired resistant genes from other Gram-negative bacteria over the years. For instance, PFGE was used to investigate the epidemiology of strains P. multocida isolated from two cases of fowl cholera outbreaks in the region of eastern Hungary. The results showed high genetic relatedness among the isolates and suggested that the second outbreak was recurrent of the same strain of P. multocida (Kardos and Kiss 2005).

MLST typing is the gold standard typing method for P. multocida (Subaaharan et al 2010). Five isolates were typed using MLST, and three STs were detected, namely, ST129 from three isolates, ST231, and ST355, as shown in table 1. Besides, ST355 was identified for the first time in the MLST database in this study. Interestingly, ST129 was recently reported in several epidemiological studies in Asia. For instance, a study was published in 2013 in China reported many outbreaks of fowl cholera involving P. multocida ST129 ( $\mathrm{n}=40$ ) (Wang et al 2013). Moreover, another study in 2016 in China reported a high occurrence (91\%) of ST129 as well (Wang et al 


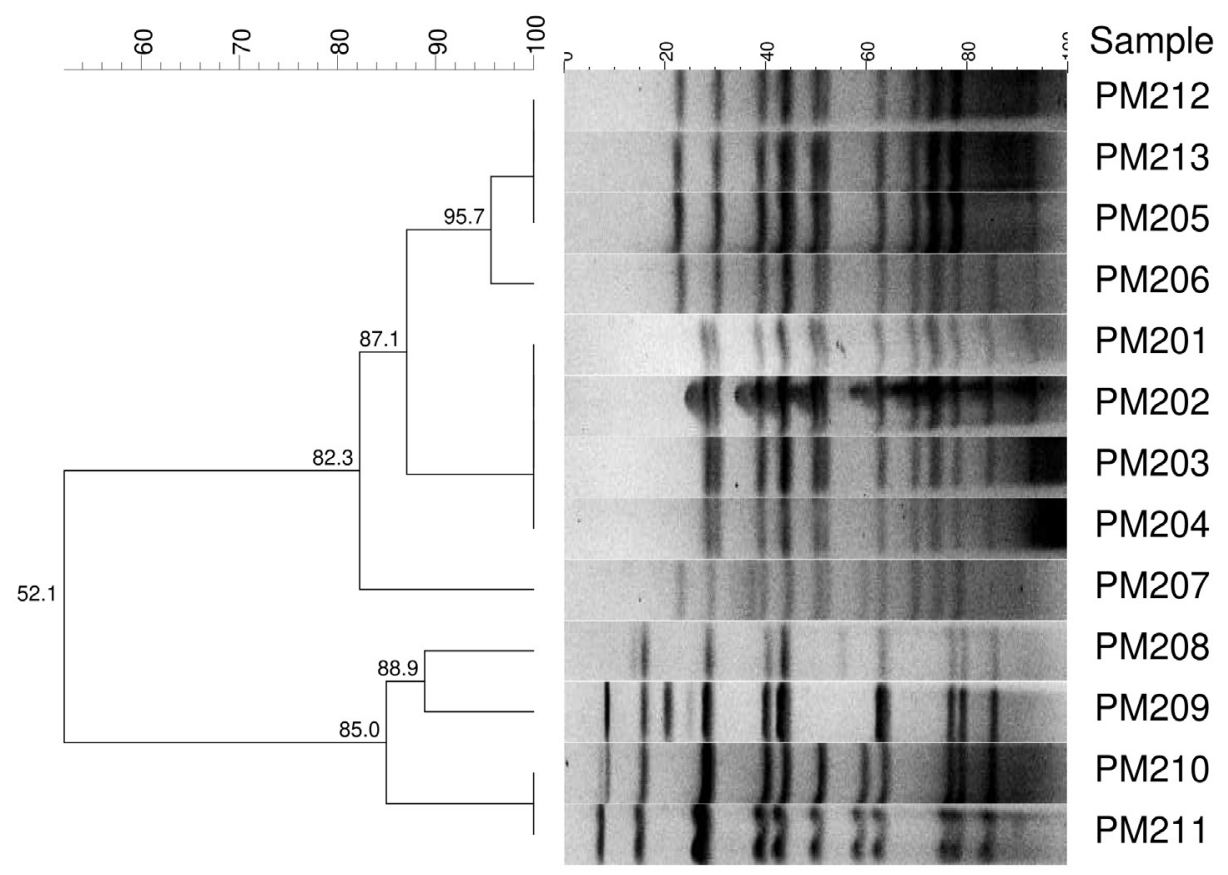

Figure 1. PFGE phylogenetic tree of P. multocida isolates using BioNumerics 6.6. The dendrogram shows high similarity (82.3\%) between the years (2000, 2013, 2014, and 2018) and high similarly among the isolates from the year 2016.

2016). A recently published study in China reported that $84 \%$ of avian P. multocida isolates were ST129, and they suggested that ST129 is a significant and high virulence ST of $P$. multocida in southwestern China, causing fowl cholera infections in many types of poultry species (Li et al 2018). The study also found that most of the ST129 isolates exhibited multidrug resistance for antibiotics, including amoxicillin, tetracycline, florfenicol, and streptomycin, which is very similar to the resistance pattern findings for the ST129 isolates in this study (table 1). Furthermore, P. multocida ST129 was also reported in India in fowl cholera outbreaks (Sarangi et al 2016). And in Iran from chicken fowl cholera outbreak according to the RIRDC MLST database ${ }^{2}$. On the other hand, the ST129 was also reported to cause hemorrhagic septicemia in bovine in Sri Lanka (Hotchkiss et al 2011), as well as infection in pigs according to the RIRDC MLST database ${ }^{3}$. As a result, ST129 was suggested as adaptable to many types of hosts (Hotchkiss et al 2011, Peng et al 2018). Although the distribution of the ST129 was discovered in India, it was suggested as a result of possible transmission from China and Sri Lanka (Sarangi et al 2016). In the current study, ST129 was identified in several fowl cholera cases among poultry in Malaysia and had a relatively similar

2 https://pubmlst.org/bigsdb?page=info\&db=pubmlst_pmultocida_isolates\&id=984

3 https://pubmlst.org/bigsdb?db=pubmlst_pmultocida_isolates \&l=1\&page=profiles antibiotic profile to the ST129 isolates reported in southwestern China (Li et al 2018). This finding shows the high possibility of transmission of ST129 from or to China into Malaysia. Besides, P. multocida was proved to spread between different countries. In a study of the fowl cholera outbreaks in Denmark and Sweden, it shows that migrating birds had spread a strain of P. multocida, which caused several fowl cholera outbreaks among these countries (Christensen and Bisgaard 2000, Petersen et al 2001). Regarding our study, Malaysia is a significant winter home for many species of migratory birds coming from the north due to its stable weather (DeCandido et al 2004). Thus, increasing the possibility of transmission of new strains into the country.

MLST is an effective tool to study $P$. multocida genotype variation and evolution (Subaaharan et al 2010). Pasteurella multocida ST231 belongs to the CC129 and shared six alleles (est, pmi, Zwf, $m d h, g d h, p g i$ ) with ST129 single locus variant (SLV) besides sharing a relatively similar antibiogram (table 1), which suggested that it evolved from ST129. Another study had published a complete genome sequence of $P$. multocida serotype A, isolated from haemorrhagic septicemia infection in buffaloes in Malaysia under the accession No. CP007205 in NCBI GenBank (Jabeen et al 2017). Based on the analysis of the RIRDC MLST database, this strain belongs to ST201, which also belongs to CC129. This isolate is SLV with ST129, this finding suggested that it evolved from ST129 and caused haemorrhagic septicemia infection in buffaloes 
in Malaysia. Haemorrhagic septicemia was reported to be endemic and of significant economic importance in many countries, including Malaysia (Benkirane and De Alwis 2002). The investigation provides significant insights into the epidemiological importance of CC129 in Malaysia. In contrast, recently published in China, there is a report on a double-locus variant (DLV) genotype from the CC129 identified as the first hypervirulent and multi-antimicrobial resistant avian P. multocida (ST342) (Zhu et al 2020). In India, SLV from CC129 (ST280) was also reported causing fowl cholera outbreaks (Sarangi et al 2016). These findings highlight the threat of $P$. multocida $\mathrm{CC} 129$ as ubiquitous and causing infections in many animal host species in Asian countries.

Overall, this study provides important epidemiological data on the diversity of $P$. multocida causing fowl cholera infection in Malaysia and highlighted the high potential of transmission of the same sequence type among the nearby countries. This study also reported the CC129 is a big threat to the poultry industry in Malaysia, and as a wildly reported CC causing fowl cholera in south Asian countries. The high antibiotic resistance shown among isolates in this study warrants the prudent use of antimicrobial agents in the poultry production in Malaysia.

\section{REFERENCES}

Arumugam N, Ajam N, Blackall P, Asiah N, Ramlan M, et al. 2011. Capsular serotyping of Pasteurella multocida from various animal hosts-a comparison of phenotypic and genotypic methods. Trop Biomed 28, 55-63.

Benkirane A, De Alwis M. 2002. Haemorrhagic septicaemia, its significance, prevention and control in Asia. Vet Med Czech 47, 234-240.

Blackall PJ, Marks D, Fegan N, Morrow C. 1995. Characterisation of Pasteurella multocida isolated from fowl cholera outbreaks on turkey farms. Aust Vet J 72, 135-138.

Botzler RG. 1991. Epizootiology of avian cholera in wildfowl. J Wildl Dis 27, 367-395.

Christensen JP, Bisgaard M. 2000. Fowl cholera. Revue scientifique et technique. Rev Off Int Epizoot 19, 626-637.

DeCandido R, Allen D, Yosef R. 2004. Merops migration at Tanjung Tuan, Malaysia: an important spring Bee-eater migration watchsite in South-east Asia. Yamashina Choruigaku Zasshi 36, 15-21.

Furian TQ, Borges KA, Laviniki V, Rocha SL, Almeida CN, et al. 2016. Virulence genes and antimicrobial resistance of Pasteurella multocida isolated from poultry and swine. Braz J Microbiol 47, 210-216.

Glisson J, Hofacre C, Christensen JP. 2013. Pasteurellosis and other respiratory bacterial infections. In: Swayne DE, Glisson JR, McDougald LR, Nolan LK, Suarez D, Nair V (eds). Diseases of poultry. $13^{\text {th }}$ ed. Wiley- Blackwell, Ames, Iowa, USA and Oxford, UK, Pp 807-823.

Gunawardana GA, Townsend KM, Frost AJ. 2000. Molecular characterisation of avian Pasteurella multocida isolates from Australia and Vietnam by REP-PCR and PFGE. Vet Microbiol 72, 97-109.

Harper M, Boyce JD, Adler B. 2006. Pasteurella multocida pathogenesis: 125 years after Pasteur. FEMS Microbiol Lett 265, 1-10.

Hassali MAA, Yann HR, Verma AK, Hussain R, Sivaraman S. 2018. Understanding antibiotics and its use in food animals. In: Antibiotic use in food animals: Malaysia overview. Hassali MAA, Yann HR, Verma AK, Hussain R, Sivaraman S (eds). Universiti Sains Malaysia, Minden, Penang, Malaysia, Pp1-11.

Heddleston K, Watko L, Rebers P. 1964. Dissociation of a fowl cholera strain of Pasteurella multocida. Avian Dis 8, 649-657.
Hindler J, Richter S, Bernard K, Bodeis-Jones S, Castanheira M, et al. 2016. Methods for antimicrobial dilution and disk susceptibility testing of infrequently isolated or fastidious bacteria. $3^{\text {rd }} \mathrm{ed}$. Clinical and Laboratory Standards Institute, Wayne, USA.

Hotchkiss EJ, Hodgson JC, Lainson FA, Zadoks RN. 2011. Multilocus sequence typing of a global collection of Pasteurella multocida isolates from cattle and other host species demonstrates niche association. BMC Microbiol 11, 115.

Jabeen S, Yong YH, Abdullah FJF, Zakaria Z, Isa NM, et al. 2017. Complete genome sequence of Pasteurella multocida serotype A strain PMTB2. 1 isolated from buffaloes that died of septicemia in Malaysia. Genome Announc 5, e01190-01117.

Jones KH, Thornton JK, Zhang Y, Mauel MJ. 2013. A 5-year retrospective report of Gallibacterium anatis and Pasteurella multocida isolates from chickens in Mississippi. Poult Sci 92, 3166-3171.

Kardos G, Kiss I. 2005. Molecular epidemiology investigation of outbreaks of fowl cholera in geographically related poultry flocks. J Clin Microbiol 43, 2959-2961.

Khoo E, Khoo L, Noormah M, Zamila Z, Nafizah M, et al. 2017. Capsular serogroup of Pasteurella multocida isolated in VRI, Malaysia from year 2014 to 2016. Malays J Vet Res 8, 60-66.

Li Z, Cheng F, Lan S, Guo J, Liu W. 2018. Investigation of genetic diversity and epidemiological characteristics of Pasteurella multocida isolates from poultry in southwest China by population structure, multi-locus sequence typing and virulence-associated gene profile analysis. J Vet Med Sci 80, 921-929.

Michael T, Sweeney MS. 2013. Performance standards for antimicrobial disk and dilution susceptibility tests for bacteria isolated from animals. $4^{\text {th }}$ ed. Clinical and Laboratory Standards Institute, Wayne, USA.

Murray BE. 1992. Problems and dilemmas of antimicrobial resistance. Pharmacotherapy 12, 86S-93S.

Nafizah M, Roseliza R, Khoo E, Khoo L, Rosnah Y et al. 2014. Prevalence of pasteurellosis from various animals species diagnosed in VRI from year 2009 to 2013. Malays J Vet Res 5, 216-217.

Peng Z, Liang W, Wang F, Xu Z, Xie Z, et al. 2018. Genetic and phylogenetic characteristics of Pasteurella multocida isolates from different host species. Front Microbiol 9, 1408.

Petersen KD, Christensen JP, Permin A, Bisgaard M. 2001. Virulence of Pasteurella multocida subsp. multocida isolated from outbreaks of fowl cholera in wild birds for domestic poultry and game birds. Avian Pathol 30, 27-31.

Sarangi LN, Thomas P, Gupta SK, Kumar S, Viswas KN, et al. 2016. Molecular epidemiology of Pasteurella multocida circulating in India by multilocus sequence typing. Transbound Emerg Dis 63, e286-e292.

Sellyei B, Thuma Á, Volokhov D, Varga Z. 2017. Comparative analysis of Pasteurella multocida isolates from acute and chronic fowl cholera cases in Hungary during the period 2005 through 2010. Avian Dis 61, 457-465.

Singh R, Blackall PJ, Remington B, Turni C. 2013. Studies on the presence and persistence of Pasteurella multocida serovars and genotypes in fowl cholera outbreaks. Avian Pathol 42, 581-585.

Subaaharan S, Blackall LL, Blackall PJ. 2010. Development of a multilocus sequence typing scheme for avian isolates of Pasteurella multocida. Vet Microbiol 141, 354-361.

Townsend KM, Frost AJ, Lee CW, Papadimitriou JM, Dawkins HJ. 1998. Development of PCR assays for species- and type-specific identification of Pasteurella multocida isolates. J Clin Microbiol 36, 1096-1100.

Townsend KM, Boyce JD, Chung JY, Frost AJ, Adler B. 2001. Genetic organization of Pasteurella multocida cap Loci and development of a multiplex capsular PCR typing system. J Clin Microbiol 39. 924-929.

Van Belkum A, Tassios PT, Dijkshoorn L, Haeggman S, Cookson B, et al. 2007. Guidelines for the validation and application of typing methods for use in bacterial epidemiology. Clin Microbiol Infect 13, 1-46.

Wang Y, Zhu J, Lu C, Wu B, Liu D, et al. 2013. Evidence of circulation of an epidemic strain of Pasteurella multocida in Jiangsu, China by multi-locus sequence typing (MLST). Infect Genet Evol 20, 34-38. 
Wang L, Sun J, Guo D, Cao P, Liu J, et al. 2016. Identification of capsule serotype and genotype of Pasteurella multocida in some areas of China. Chin J Prev Vet Med 2, 116-119.

Wilkie IW, Harper M, Boyce JD, Adler B. 2012. Pasteurella multocida, diseases and pathogenesis. In: Aktories K, Orth J, Adler B (eds). Current topics in microbiology and immunology. Springer, Berlin, Pp 1-22.
Wilson BA, Ho M. 2013. Pasteurella multocida: from zoonosis to cellular microbiology. Clin Microbiol Rev 26, 631-655.

Zhu D, Yuan D, Wang M, Jia R, Chen S, et al. 2020. Emergence of a multidrug-resistant hypervirulent Pasteurella multocida ST342 strain with a floR-carrying plasmid. J Glob Antimicrob Resist 20, 348-350. 